\title{
Efectos psicológicos de la relación con pacientes en la persona del clínico*
}

Jozef Corveleyn

Universidad Católica de Lovaina

Lovaina, Bélgica

El artículo presenta diversas interpretaciones psicoanalítico-humanistas que se hacen en la literatura científica con respecto a los efectos que la acción terapéutica tiene sobre el psicólogo clínico, en él como persona, y en sus relaciones con los pacientes. Se discute la contribución de M.B. Sussman sobre la identidad de la psicoterapia profesional, la motivación para ser terapeuta y las gratificaciones y desventajas de esta actividad. Entre otros aportes se afirma que los procesos contratransferenciales pueden tener dos aspectos: neuróticos y existenciales positivos, y se sugiere especial cuidado en la formación de psicoterapeutas para evitar hacer daño tanto a los pacientes, como a sí mismos.

psicólogo clínico como persona / contratransferencia / bienestar psicológico

\section{Psychological effects of the relations with patients on the clinical psychologist as a person}

This paper introduces different psychoanalytic-humanist interpretations related to the effects of therapeutic actions on the clinical psychologist as a person and in his/her relations with patients. Sussman's contributions on the identity of the professional therapist, the motivations to become one and the rewards and disadvantages of the profession are discussed. The autor argues that countertransferences in the therapeutic process have two aspects: neurotic and positive-existentialist.

clinic psychologist as a person / countertransference / psychological well-being

* Versión en español editada por Ricardo Braun y José Cuny. 
Ningún psicólogo clínico que trabaje como psicoterapeuta o psicometrista, o que realice una tarea de coordinación o animación de tratamiento grupal, podrá escapar a la experiencia de que su trabajo exige mucho de sí mismo, llegando incluso en ocasiones a comprometer su vida personal. Esto puede resultar muy agotador. Sin embargo, existe en cambio la posibilidad de que el clínico disfrute intensamente con el progreso de sus pacientes, el psicometrista encuentre satisfacción en sus claras y psicológicamente lúcidas construcciones sobre los problemas de la persona a quien examinó con gran esfuerzo, y el psicólogo del grupo terapéutico esté muy satisfecho de la buena atmósfera reinante en éste.

El contraste entre las formas de influencia del trabajo clínico en la persona del propio clínico no es coincidencia. Pero a pesar de su importancia no se le presta mayor atención en la literatura científica general. En la literatura psicoterapéutica sí, aunque en ésta con mucha frecuencia se lee que es preferible que el terapeuta no se deje "llevar" en su contacto con los pacientes. Se sugiere que sea neutral, objetivo, distanciado, profesional, etc. Sin embargo, a partir de los años ochenta esta tendencia ha ido cambiando, fomentando en el clínico actitudes más positivas: menos ansioso, más abierto y menos defensivo.

En esta contribución me aproximaré a la problemática de la influencia del tra- bajo clínico en el propio clínico, principalmente desde una perspectiva psicoterapéutica.

\section{EL "INSTRUMENTO BÁSICO": LA PERSONA DEL CLÍNICO}

En la mayoría de los cursos y manuales de psicoterapia se ha subrayado siempre la importancia de la persona del terapeuta para la plena realización del proceso terapéutico. Esto mismo podría extenderse a todo el amplio panorama de la práctica en psicología clínica. En realidad, el instrumento más importante con que cuenta el clínico en su trabajo con sus pacientes es su propia persona.

Su trabajo es, en esencia, un diálogo, un acontecimiento intersubjetivo. Ni la terapia ni la investigación diagnóstica son procesos técnicos impersonales y neutrales en los que el terapeuta se encuentra frente a una realidad radical y absolutamente diferente y distante. La implementación terapéutica requiere de la participación activa del paciente y de la verdadera interacción entre éste y el terapeuta. En esa interacción los dos son equivalentes, al punto de poderse cuestionar que la actividad del clínico sea el factor más importante en la realización de los procesos de cambio previstos. Considero que, en esencia, el terapeuta no es el verdadero iniciador de esos procesos. Más bien, es su mediador o facilitador. Los cambios terapéuticos en el paciente son, finalmente, el resultado de su propia actividad. 
Ahora bien, para poder cumplir con su rol facilitador, el clínico, en tanto persona, debe insertarse en un diálogo con el paciente apreciado como cosujeto. Tengamos presente que toda su personalidad llega a ser interpelada y dirigida en esa conversación con la persona que recurre a su pericia como facilitador. Hay que señalar que muchos factores personales entran en conflicto en este proceso. No sólo intervienen los llamados factores personales objetivos, es decir, los relacionados con las habilidades terapéuticas y otras capacidades - destrezas (habilidades de contacto aprendidas), conocimiento (teoría de terapia y técnicas) y la experiencia (conocimiento adquirido en la rutina diaria y en el ejercicio profesional)-. También intervienen los factores personales subjetivos: la propia historia del terapeuta y, relacionado con ello, la motivación personal para llegar a ser terapeuta. Las investigaciones recientes muestran una mayor atención a la vida del terapeuta, a su sistema de valores y a su forma de dar sentido a la existencia (Bergin \& Jensen, 1990; Beutler et al., 1994).

\section{Problemas de identidad}

El hecho de que se haya escrito tan temerosamente acerca de la persona del terapeuta se puede atribuir a la juventud de la psicoterapia profesional. Considerar aún la psicología, que tiene más de cien años, como una ciencia nueva, es a lo mejor un poco exagerado, pero no lo es en el caso de la psicoterapia profesional a la que se puede reconocer como una profesión nueva. Recordemos que hasta hace poco esta nueva profesión estaba sobre todo preocupada con problemas referidos a su propia identidad.

Por un lado, tenía que demarcar su terreno específico de investigación dentro de la propia familia científica de la psicología. Por mucho tiempo el campo psicoterapéutico estaba casi completamente dominado por la escuela psicoanalítica. A partir de los años cincuenta otras tendencias terapéuticas se fueron constituyendo poco a poco en parte importante de su campo, aunque bajo diferentes formas de relación con la psicología "establecida" y la psicología académica. Así, nacieron sucesivamente la tendencia humanista y experiencial, la escuela conductual y las terapias sistémicas de familia, para nombrar sólo las más importantes. Es evidente que el proceso de definición y demarcación interna del terreno de la psicoterapia y de ésta en el conjunto de la psicología en general, ha exigido la inversión de mucho esfuerzo.

Por otro lado estaba la lucha de emancipación externa, particularmente de la psiquiatría médica que tradicionalmente ocupó el terreno de la salud mental. Las investigaciones sobre la identidad y la eficiencia de las psicoterapias absorbieron innegablemente caudales de energía durante numerosos años. Otros esfuerzos se dirigieron 
también a la defensa de la importancia de la psicoterapia en el contexto profesional general y a la creación de un clima de confianza en el gran público. Estos motivos explican la razón por la que se ha distraído la atención de los problemas internos y más íntimos de los que ejercen esta nueva profesión. Problemas de juventud que, por lo demás, hacen muy comprensible toda clase de tendencias de autoprotección.

\section{Investigación motivacional}

Un ejemplo interesante de la tendencia reciente a poner atención en la persona del terapeuta, es la investigación acerca de los motivos por los que la gente elige ser psicoterapeuta, o en su caso, sigue ejerciendo esta difícil profesión durante una carrera completa. Tal como ocurre en la elección de cualquier profesión, seguramente se podrá encontrar en los psicoterapeutas toda clase de motivos (subconscientes), algunos tal vez patológicos relacionados con la historia personal. Complementariamente, cabría preguntarse si es posible sostener que en la elección de la profesión intervienen patrones de motivación consistentes y más positivos. ¿Es esta profesión gratificante para aquéllos que la practican?

Sobre este tema no existen muchas investigaciones (véase el corto sumario de Beutler et.al., 1994), aparte del estudio de Sussman (1992), que fue publicado recientemente bajo el título $\mathrm{A} \mathrm{cu}$ rious calling.
La investigación de Sussman hace eco del consenso de la literatura sobre el hecho de que uno de los determinantes más fuertes de la elección de la psicoterapia como profesión es el deseo (inconsciente) de resolver los propios problemas emocionales. Con eso se refiere a algunos conflictos psicológicos típicos. La investigación demuestra que cuando se presta la suficiente atención a estas dificultades durante la formación del terapeuta, éstas no sólo no causan daño alguno, sino que, por el contrario, mejoran la capacidad de los psicoterapeutas para ayudar a sus pacientes. El poner suficiente atención a esas dificultades no sólo exige una terapia personal durante la enseñanza especializada, sino también suficiente entrenamiento cognitivo y una forma de cuidado permanente a través de la supervisión durante toda la carrera, entre otras acciones.

La parte más extensa del estudio de Sussman está dedicada a aquellos conflictos psicológicos muchas veces inconscientes. De acuerdo a Sussman, éstos son la fuente de motivación más importante para el psicoterapeuta.

\section{El propio fondo personal}

Con respecto al tema de la interioridad personal profunda de los psicoterapeutas y sus conflictos psicológicos más inconscientes, me referiré particularmente a los datos referidos a su propio fondo familiar.

Sobre estos datos hay en la literatura bastante controversia, puesto que no 
hay un consenso sobre la presencia de patrones específicos. Algunos autores señalan que en la historia de los terapeutas existe una marcada situación de estrés emocional en su familia de origen. Es como si ellos desde pequeños hubieran sido entrenados para experimentar y manejar condiciones emocionales muy difíciles. Otros indican también que, con frecuencia, muchos terapeutas en su juventud tuvieron que vivir afectivamente muy aislados como consecuencia de la pérdida prematura de uno de los padres, o de la posición social marginal de estos últimos frente al mundo exterior.

Aparecen dos patrones sistémicos familiares dominantes: 1) el futuro terapeuta tuvo en su familia de origen el rol de cuidarla, y así se acostumbró a acoger siempre a los que sufren; y 2) el futuro terapeuta jugó siempre el rol de "mediador" entre padres emocionalmente inestables y el grupo de hijos. Sussman considera que estos patrones están relacionados con dos dificultades identificables en numerosos terapeutas: algunos han estado tan habituados a fijarse de inmediato en las necesidades emocionales de los demás que, luego en su propia vida (incluida la profesional) tienen dificultades para poner suficiente atención a sus propias necesidades emocionales; otros han aprendido a suprimir su propia agresión con tal intensidad que, incluso en su trabajo profesional, no son capaces de protegerse de manera suficiente contra situaciones potencialmente negativas asociadas a la relación con sus pacientes.

Asimismo, los investigadores sostienen que muchos terapeutas muestran, por la influencia de aquellas experiencias prematuras y juveniles, una diligencia exagerada para "salvar" a sus pacientes. Sussman $(1992,1995)$ opina que uno de los dos siguientes patrones inconscientes de motivación pueden jugar un rol en ello. En algunos casos se trata de un sentimiento inconsciente de culpa por el daño, real o imaginario, que ellos pudieron haber causado a sus seres queridos o que potencialmente pudieran causar. Por eso están dispuestos a hacer enormes sacrificios en su vida profesional para disminuirlo creando condiciones de reparación a través de la relación con sus pacientes. En otros casos se trata de terapeutas que han entrado en el campo de la psicoterapia movidos por la intención de luchar contra la ansiedad generada por un trastorno psíquico que los lleva finalmente a preocuparse por los problemas de los demás. Esta ansiedad (inconsciente) tiene su raíz en las experiencias patológicas que vivieron desde pequeños en su propia familia.

\section{Gratificaciones del terapeuta}

Se constata además, con facilidad, que en el psicoterapeuta existen motivos más conscientes y de acceso más fácil vinculados con la realización de ciertas ambiciones y la obtención de diversas gratificaciones relacionadas con 
su profesión. Sobre esto se habla muy poco. Pero se trata de factores muy reales (Sussman, 1992). Así, tenemos las gratificaciones más intrínsecas asociadas, por ejemplo, a la sensación de hacer un trabajo útil para los demás o de ser indispensable para ellos, o al placer que a veces uno puede sentir no sólo por entender sino también ayudar a otros a dominar situaciones humanas muy conflictivas, o al saber que uno puede ayudar a la gente a enriquecerse personalmente. En todo esto el terapeuta puede experimentar que él llega a ser más fuerte, que su desarrollo personal continúa y que él en el contacto con sus pacientes encuentra la oportunidad de una afirmación propia y recíproca. Hay además otras formas más extrínsecas de satisfacción profesional, por ejemplo, el placer del continuo contacto humano no específico, el escapar a la soledad, el llegar a una posición socialmente apreciada, etc. A éstas hay que agregar las satisfacciones menos bellas, más neuróticas, como la del terapeuta que no solucionó sus propios problemas juveniles de autoridad y que encuentra placer par personne interposée en actuar sus pensamientos rebeldes; o la de aquel otro que en su trabajo busca la realización de fantasías narcisísticas todopoderosas.

En todos estos casos, la pregunta crucial es, por supuesto, hasta qué punto está permitido que el terapeuta encuentre satisfacciones psicológicas personales en su trabajo con pacientes. La res- puesta debe precisarse. El sugerir que él no debe buscar ni encontrar en su trabajo satisfacción alguna no es muy realista. Simplemente, no habrían terapeutas. Es normal que él como muchas otras personas busque y encuentre satisfacción en su trabajo. Por ello es difícil señalar en forma precisa la frontera de lo permisible o recomendable. El hecho de que algunas satisfacciones profesionales puedan devenir en situaciones de abuso del paciente no justifica que uno deba tomar en esta materia una actitud particularmente fóbica. Stierlin (1972) señala dos formas de exageración con respecto a la relación con los pacientes: terapeutas que necesitan intensamente a sus pacientes para su propia realización y que terminan por explotarlos, propiciando su dependencia por mucho tiempo; y terapeutas que al contrario necesitan muy poco a sus pacientes en términos de satisfacción personal, por lo que se hallan restringidamente motivados para invertir en ellos su mayor capacidad de trabajo y la amplitud de empatía requerida. Que el terapeuta en su trabajo intente satisfacer sus propias necesidades, repito, es algo normal e inevitable. Pero hay que evitar que las motivaciones enunciadas anteriormente, personales y sobre todo las intrínsecamente neuróticas, vayan a hacerse de un lugar preponderante de modo que el quehacer psicoterapéutico sirva sobre todo para satisfacerlas. 


\section{Pasado el tabú}

La reciente investigación de Sussman indica que se encuentra en desarrollo una actitud de apertura frente al análisis y estudio de la persona del psicoterapeuta. Mientras que en la temprana época posfreudiana no era siquiera un tema de estudio, ahora se le puede discutir abiertamente.

\section{Cambio de clima}

El cambio de clima que se observa resulta de una actitud más transparente en la percepción de la personalidad del terapeuta, especialmente desde los años setenta en que se habla con gran apertura de las vulnerabilidades y limitaciones humanas de los terapeutas, $\mathrm{y}$ de las consecuencias que ellas ocasionan sobre el éxito de la terapia. Se efectúan serias investigaciones en casi todas las facetas de esta problemática: investigaciones sobre formas leves o menos leves de psicopatología padecidas por los psicoterapeutas; sobre las motivaciones conscientes o inconscientes que los animan; y sobre los antecedentes familiares tanto de los psicoterapeutas, como de los candidatos a una formación en psicoterapia y de las personas que participan en dicha formación. También se evalúan los beneficios de la psicoterapia personal, los diversos aspectos psíquicos que pueden jugar un rol en el síndrome de agotamiento, y las circunstancias psicosociales que promueven o mantienen el abu- so en la relación psicoterapéutica. Realmente, ninguna faceta resulta desatendida (Beutler et al., 1994). También en trabajos recientes de tipo divulgación sobre psicoterapia, se tocan muchos de los temas nombrados (Strean \& Freeman, 1989; Yalom, 1989).

\section{Historia reciente}

Para la quiebra de esta especie de tabú en torno a la participación de la persona del terapeuta han intervenido al menos tres factores históricos importantes.

En primer lugar, la conclusión presente en muchas investigaciones de que los factores llamados a-específicos definen de manera muy importante el éxito de una psicoterapia, con seguridad bastante más que los factores específicos ligados a una teoría y a una técnica. Esto significa, entre otras cosas, que la persona del terapeuta es más importante que las técnicas que utiliza y que las teorías que profesa y afirma aplicar (véase Bergin \& Garfield, 1994).

Luego se puede señalar, sobre todo después de los años setenta, el hecho de un cambio sustancial en la corriente principal del pensamiento psicoanalítico respecto de la llamada contratransferencia. Esta evolución ha ejercido una gran influencia en el panorama general de la psicoterapia. La contratransferencia se refiere, en principio, a la reacción emocional global que el encuentro con 
el paciente provoca en el terapeuta. Se reconoce no sólo que el fenómeno proviene de una relación psicoterapéutica intensa e íntima sino además que en cada contacto clínico la contratransferencia juega un rol importante (véase, para un sumario general, Wolstein, 1998 y Epstein \& Feiner, 1979).

En la contratransferencia se puede diferenciar dos aspectos: por un lado un componente neurótico y repetitivo; por otro el componente de cooperación, contacto y simpatía humana básica por el paciente. En la literatura psicoanalítica clásica se ponía atención, exclusivamente, en los peligros del primer componente. Por este hecho, entre otros, se acentuaba, con razón, la necesidad de una terapia personal (análisis didáctico) para el analista. Una corriente más reciente, sin negar la importancia del componente neurótico, pondrá su mayor atención en el segundo componente. En esta visión la contratransferencia no será sólo apreciada como un posible peligro para la terapia, sino también como una modalidad profunda de comunicación con el paciente fomentada por la terapia misma, que puede y debe ser usada por el terapeuta como auxiliar para lograr interpretaciones óptimas, que hacen avanzar el proceso (Lane \& Storch, 1986; Corveleyn, 1989). Este enfoque ha determinado una muy amplia apertura respecto del rol que le corresponde a la persona del terapeuta en esta "profesión imposible". Así, se llegan a debatir abiertamente, por ejem- plo, las dificultades asociadas con la contratransferencia (por ejemplo, el caso histórico Jung/Spielrein: Carotenuto, 1983 y Kerr, 1994; y en general: Strean, 1986; Giovacchini, 1989) y la necesidad de preservar un interés benévolo bien dosificado del terapeuta por el paciente (véase Kohut, 1959; Greenson, 1960; Poland, 1975), como Freud por lo demás ya había mostrado de modo explícito en su trabajo clínico (véase por ejemplo Dorpat, 1977; Lane \& Storch, 1986).

Finalmente, hay que referirse a un factor muy reciente, la seria preocupación en el mundo contemporáneo de la psicoterapia profesional por la problemática del abuso sexual o de otro tipo, en la relación terapéutica (véase sobre todo Pope et al., 1993; Pope, 1994). Existen en este contexto no sólo investigaciones de incidencia y prevalencia, sino también importantes investigaciones sobre las necesidades y posibilidades de prevención (véase sobre todo Pope, Sonne \& Holroyd, 1993 y Jehu, 1994). Con respecto a esto, se remarca la importancia de la terapia personal en la formación del futuro psicoterapeuta; que reciba información adecuada sobre los múltiples componentes emocionales que intervienen en la relación terapéutica, cualquiera sea su encuadre teórico-práctico. Así, se pone cada vez mayor énfasis en romper el aislamiento y soledad en que se hallan aparentemente muchas veces los potencialmente "abusivos". Para evitar que lo inad- 
misible brote y se instale se requiere sobre todo apertura en el mismo grupo profesional de psicoterapeutas: los "abusivos" potenciales deben poder acceder a sus supervisores y colegas de modo que sus problemas no sean tratados cuando sea ya demasiado tarde. Estos terapeutas tienen que aprender por sí mismos a reconocer, rápidamente, las señales de posibles conductas propias de abuso, y deben poder encontrar, especialmente en su propio grupo de enseñanza, un camino abierto para la ayuda y el seguimiento adecuados. Por encargo de la Asociación Psicológica Americana, Pope (1994) ha formulado propuestas muy concretas acerca de la búsqueda (self-assessment), terapia y acompañamiento didácticos. Todo esto hace necesario una visión y disposición muy amplias sobre el problema planteado en general, junto, claro está, a la discreción frente al caso individual.

Nació así progresivamente la siguiente atmósfera: en vez de evitar ansiosamente el problema de las implicancias posibles del terapeuta en su labor profesional, manteniéndolo así escondido para el debate público, se ha acrecentado cada vez más la tendencia a enfrentarlo de manera lúcida. Esto es ciertamente favorable para las posibilidades de desarrollo tanto de los pacientes como de los terapeutas.

\section{El TERAPEUTA EN EL ASIDERO DE SU PROPIO TRABAJO}

El ejercer psicoterapia tiene una gran influencia en la persona del terapeuta.
Eso está fuera de toda duda, como también lo está el que esa influencia tiene lugar dentro y fuera de las horas de trabajo.

\section{Contratransferencia}

La forma e intensidad en que la historia del paciente influye en la persona del terapeuta -fenómeno de contratransferencia- se determina por la orientación asumida por las partes (paciente-terapeuta) en su interacción. Por un lado está la estructura de personalidad del terapeuta con sus características y antecedentes personales -que incluyen componentes neuróticos y repetitivos que tienen que ser socorridos terapéuticamente-, su educación, experiencia y fondos teóricos, que definen el color y naturaleza del eco que el terapeuta puede dar a la historia del paciente. Y por otro lado está también la manera de ser del paciente que constituye un factor intensamente determinante para la influencia de la terapia en el propio clínico.

El contrato terapéutico no representa una cuestión de neutralidad en la relación terapeuta-paciente. Representa más bien el establecimiento de un vínculo formal de cooperación entre ellos, adaptado a la naturaleza muy especial de la relación terapéutica y las características psíquicas del paciente. Cada clínico sabe, por experiencia, de la diferencia existente entre una terapia con un paciente limítrofe con riesgo de suicidio, que tiene muchas dificultades 
para respetar los límites terapéuticos y la terapia con un paciente joven levemente neurótico, molesto por su inseguridad y sentimientos de inferioridad. Aquí hay una diferencia objetiva: en el primer caso, el terapeuta experimentará en sí mismo más ansiedad e inseguridad, y a veces también irritación por la presión afectiva permanente. En el segundo caso él podrá trabajar menos tenso pudiendo ofrecerle al paciente todo el espacio que requiera el diálogo para que trate de conquistar sus inhibiciones de manera autónoma. Es evidente que las diferencias que se observan en una situación frente a la otra, sobre el plano contratransferencial, no serán atribuibles, en primer lugar, a la personalidad del terapeuta, sino más bien a la influencia -objetivadel paciente. Éste es el caso de la llamada "contratransferencia objetiva" (véase, entre otros, Kernberg, 1975).

Considero que esta nueva dimensión de la contratransferencia, que se agrega a las antes referidas -el componente neurótico y el interés básico por el paciente- debe ser tomada muy en cuenta durante la enseñanza. El terapeuta tiene que aprender a controlar y manejar las diferentes formas de presión psíquica a que se ve sometido por los pacientes y sus características patológicas. Pero en realidad ese aprendizaje no debe limitarse al tiempo de la formación, pues uno no queda preparado para todo. Con cierta frecuencia es necesario buscar después, ya en el ejerci- cio profesional, suficientes posibilidades para una deliberación compartida, "colegiada", en particular aunque no únicamente, cuando uno trabaja con pacientes con problemas que provocan intensa presión afectiva.

En la literatura más reciente acerca del exigente trabajo psicoterapéutico con ciertos grupos específicos, las medidas relatadas son consideradas como las únicas eficientes. Me refiero por un lado a la literatura de testimonio: Tick (1995) en su trabajo con veteranos de guerra; Shernoff (1995) en su acompañamiento psicoterapéutico a pacientes terminales, entre otros a pacientes con sida; Jobes y Maltsberger (1995) en sus trabajos con pacientes suicidas; Steinberg y Salamon (1995) en su trabajo psicoterapéutico con pacientes geriátricos. Por otro lado me refiero también a numerosas investigaciones sobre el efecto muy acentuado de hacer psicoterapia con pacientes limítrofes (Nadelson, 1976; Adler, 1985) y con pacientes severamente traumatizados, caso, entre otros, de las víctimas de incesto (véase McCann \& Pearlman, 1990; Pearlman \& Saakvitne, 1995).

\section{El bienestar general del terapeuta}

El quehacer psicoterapéutico ejerce una gran influencia en el propio terapeuta, no sólo durante la terapia, también después, incluso a lo largo de toda la vida. Los aspectos más positivos de esta influencia los he mencionado en la discusión de las "gratificaciones" nor- 
males de esta profesión. Ahora debo referirme al hecho de que las investigaciones contemporáneas sobre las llamadas variables subjetivas que actúan sobre los psicoterapeutas señalan que el rol del terapeuta puede influenciar negativamente en su propio bienestar (véase Beutler et al., 1994), determinando en ellos un aumento significativo del estrés, además de otros transtornos severos, como aumento del neuroticismo, una disminución del autoaprecio, burn-out y problemas de relación (véase Guy \& Liaboe, 1986).

En una perspectiva diferente se observan interesantes investigaciones acerca de la relación entre la salud emocional general del terapeuta y el resultado terapéutico. Una salud lábil del terapeuta impide el avance del paciente (Lambert \& Bergin, 1983). Esto está demostrado tanto en investigaciones cuasiexperimentales como en investigaciones más "naturalísticas".

Se podría esperar entonces, de manera general, que los terapeutas al pasar por una terapia personal estarían poniendo remedio suficiente para ayudar a desaparecer el problema representado por el estancamiento de muchos pacientes. Esto no resulta así, tal como se pone de manifiesto en las conclusiones de la investigación empírica (datos en diversas direcciones). Muchas veces, sin embargo, se busca en estas investigaciones sólo la relación entre el dato que el terapeuta ha pasado o está pasando por una terapia personal y la efi- ciencia de la terapia que él está realizando en su paciente.

No he encontrado reportes sobre el efecto de la terapia personal en el actual o posteriormente amenazado bienestar psicológico del terapeuta. Sobre esto $\mathrm{y}$, teniendo en cuenta la influencia potencialmente negativa del ejercicio profesional de la psicoterapia en la vida del terapeuta, deberían haber más investigaciones.

En lo que se refiere a la relación entre terapia vivida por el candidato durante la formación o por el terapeuta ya formado y la eficiencia que muestren en su trabajo psicoterapéutico, se observa que en algunas ocasiones se llega a resultados positivos con los pacientes. Sin embargo, en otras ocasiones no se llega a establecer una relación significativa. Esto podría vincularse con el hecho de que el estar involucrado activamente en una terapia personal podría afectar temporalmente las habilidades y la eficiencia del terapeuta, por hallarse comprometido en una exploración intensa de su propia persona.

Pero seamos prudentes: estos hallazgos parcialmente contradictorios, no dicen nada definitivo sobre la utilidad - para paciente y terapeuta- de una terapia personal en el contexto de una formación en psicoterapia. En base a los datos de las investigaciones efectuadas, no se puede llegar a discriminar fehacientemente si el hecho de que en un momento posterior en su carrera profesional el terapeuta reporte haber 
pasado por una terapia personal, sea indicador o no de un estado mental originalmente lábil, o que se trata más bien de un estado psíquico mejorado como consecuencia de aquella terapia. Quizás en una investigación general, a terapeutas de todas las posibles tendencias académicas, aquellos que indiquen haber tenido una terapia personal, resulten ser los que menos necesitan de ella (véase Beutler et al., 1994).

\section{Conclusión}

Se ha de acreditar que, aparte del conocimiento, técnica y habilidad, la persona del clínico juega un rol central en la clase especial de trabajo que él efectúa. También, que existe una clara relación entre características personales del terapeuta, la elección que hiciera de este tipo de trabajo, el modo como lo realiza y los resultados que logra en sus pacientes, confirmándose además que el impacto de este trabajo en su persona no es pobre en significación.

Ahora bien, no todas estas formas de influencia se concretan como consecuencias negativas para los psicoterapeutas. Por el contrario, se trata de factores subjetivos que forman parte de las condiciones mismas de este trabajo y que pueden llamarse inevitables por estar intrínsecamente ligadas a la naturaleza del trabajo psicoterapéutico.

No es pues apropiado minimizar o intentar ocultar estos factores personales. Una reacción fóbica al respecto está fuera de lugar; también una actitud alarmista exagerada. Los factores personales constituyen un dato central al que se debe poner constante atención en la enseñanza, en el cuidado permanente de instituciones de formación psicoterapéutica, en las comisiones éticas de las asociaciones de profesionales y en la vigilancia personal del clínico. La prevención y el cuidado permanente deben estar siempre presentes.

Poner atención no quiere decir intentar hacer desaparecer aquellos componentes personales $\mathrm{u}$ otorgarles una aureola negativa pues no se trata de aspectos intrínsecamente equivocados. Se trata de aspectos que pueden ejercer un efecto negativo para el paciente (actividad psicoterapéutica defectuosa) como también para la vida personal del psicoterapeuta cuando no se les toma suficientemente en cuenta.

El clínico tiene que aprender a manejar aquellos aspectos ligados a su persona. Esto significa en primer lugar que tiene que aprender a conocer profundamente sus propios conflictos, motivaciones, peculiaridades de su carácter e intenciones, para evitar situaciones de riesgo ligadas a ello. No se trata aquí básicamente de un conocimiento teórico sino más bien de un conocimiento vivenciado gracias a la psicoterapia personal. Este trabajo psicoterapéutico debe realizarse en primer término durante la formación, pero si es necesario debe ser continuado sin amilanamiento más adelante durante el ejercicio de la carrera. La psicoterapia 
personal también es válida contra el estrés que provocan algunas situaciones de psicoterapia o grupos de problemas a los que el clínico se debe enfrentar. Luego, se requiere tener presente los evidentes aspectos de la enseñanza: la teoría propia de la corriente, el conocimiento técnico y las habilidades.

Finalmente, hay que destinar el tiempo necesario para supervisión e "intervisión" no sólo durante el período estrictamente de formación, sino más bien como algo permanente en lo que el clínico siempre se pueda apoyar. Acerca de esto último tendría que desarrollarse una evidencia empírica más extensa en el grupo de profesionales. Algunas investigaciones indican que sólo de esta manera se puede evitar una malformación y abuso en la actividad psicoterapéutica.

La psicoterapia personal y la permanente deliberación profesional colegiada son los medios más importantes para encontrar y conservar cierto equilibrio entre las fuerzas positivas de la propia personalidad, que hacen posible el trabajo psicoterapéutico, y las fuerzas negativas, los peligros "neuróticos", que pueden contagiar o infectar el trabajo.

Para llegar a un desarrollo equilibrado de la actividad profesional en psicología clínica, y más específicamente en psicoterapia, el clínico debe cuidar de manera suficiente un aspecto adicional: los "remedios" de naturaleza no profe- sional. Estos medios no son específicos para la empresa de la psicoterapia, pero sí aportan de manera importante a la conservación de una salud mental suficiente.

Ser psicoterapeuta puede brindar la oportunidad de una muy significativa realización de la propia vida, pero no puede llenarla, si no pasaría a ser rápidamente un "guruismo", un "supersacerdotismo" u otra ilusión mesiánica. Hacer psicoterapia es quizás una vocación -un poco "peculiar" según Sussman (1995)- pero también es una profesión, un trabajo. Y para obtener calidad en este trabajo, el psicoterapeuta debe gozar de una vida equilibrada; para ello debe mantener otras formas de relación con su ambiente y con el ambiente de sus pacientes. Así seguirá investigando con suficiente energía en aspectos personales, familiares, amicales, sociales y culturales. Porque la vida de sus pacientes nunca llega a ser totalmente definida por factores psicológicos. 


\section{REFERENCIAS}

Adler, G. (1985). Borderline psychopathology and its treatment. Northvale, N.J. London: Jason Aronson.

Aghassy, G. \& Noot, M. (1987). Seksuele contacten binnen psychotherapeutische relaties [Sexual contacts in the context of psychotherapeutic relationships]. Tijdschrift voor Psychotherapie [Journal of Psychotherapy], 13, 293-337.

Bergin, A.E. \& Jensen, J.P. (1990). Religiosity of psychotherapists: A national survey. Psychotherapy: Journal of the Division of Psychotherapy, American Psychological Association, 27, 3-7.

Bergin, A.E. \& Garfield, S.L. (Eds.) (1994). Handbook of psychotherapy and behavior change. New York: John Wiley \& Sons.

Beutler, L.E., Machado, P.P. \& Allstetter, S. (1994). Therapist variables. En A.E. Bergin \& S.L. Garfield (Eds.), Handbook of psychotherapy and behavior change. New York: John Wiley \& Sons.

Carotenuto, A. (1983). A Secret symmetry: Sabina Spielrein between Freud and Jung. New York: Pantheon.

Corveleyn, J. (1989). Over tegenoverdracht gesproken: hinderpaal of hulpmiddel? [Sobre contratransferencia: ¿impedimento o ayuda?]. En H. Vertommen, G. Cluckers \& G. Lietaer (Eds.), De relatie in therapie [The relationship in therapy] (Studia psychologica) (pp. 103-119). Leuven: Universitaire Pers Leuven.
Dorpat, T.H. (1977). On neutrality. International Journal of Psychoanalytic Psychotherapy, 6, 39-64.

Epstein, L. \& Feiner, A.H. (Eds.) (1979). Countertransference. New York/ London: Jason Aronson

Foudraine, J. (1971). Wie is van hout... [Who is made of wood?...]. Bilthoven: Ambo.

Giovacchini, P.L. (1989). Countertransference: Triumphs and catastrophes. Northvale, N.J./ London: Jason Aronson.

Greenson, R.R. (1960). Empathy and its vicissitudes. International Journal of Psychoanalysis, 41, 418-424.

Guy, J.D. \& Liaboe, G.P. (1986). The impact of conducting psychotherapy on psychotherapists interpersonal functioning. Professional Psychology: Research and Practice, 17, 111-114.

Jehu, D. (1994). Patients as victims: Sexual abuse in psychotherapy and counselling. New York: John Wiley \& Sons.

Jobes, D.A. \& Maltsberger, J.T. (1995). The hazards of treating suicidal patients. En M.B. Sussman (Ed.), A perilous calling: the hazards of psychotherapy practice (pp. 188-199). New York: John Wiley \& Sons.

Kernberg, O. (1975). Borderline conditions and pathological narcissism. Northvale, N.J./ London: Jason Aronson.

Kerr, J. (1994). A most dangerous method: the story of Jung, Freud and Sabina Spielrein. New York: Vintage Books.

Kohut, H. (1959). Introspection, empathy and psychoanalysis. Journal of the American Psychoanalitic Association, 7, 4-59. 
Lambert, M.J. \& Bergin, A.E. (1983). Therapists characteristics and their contribution to psychotherapy outcome. En C.E. Walker (Ed.), The handbook of clinical psychology (Vol. I). Homewood, Il: Dow Jones-Irwin.

Lane, R.C. \& Storch, R.S. (1986). A fortuitous extra-analytic event: countertransference, hindrance or benefit? En H.S. Strean (Ed.), Countertransference. Current Issues in Psychoanalytic Practice (Vol. 2). New York/London: Haworth Press.

McCann, L. \& Pearlman, L.A. (1990). Vicarious traumatization: a framework for understanding the psychological effects of working with victims. Journal of Traumatic Stress, 3, 131-149.

Nadelson, T. (1976). Victim, victimizer: interaction in the psychotherapy of border-line patients". International Journal of Psychoanalytic Psychotherapy, 5, 115-129.

Pearlman, L.A. \& Saakvitne, K.W. (1995). Trauma and the therapist: countertransference and vicarious traumatization in psychotherapy with incest survivors. New York/London: W.W. Norton \& Company.

Poland, W.S. (1975). Tact as a psychoanalitic function. International Journal of Psychoanalysis, 56, 155-162.

Pope, K.S. (1994). Sexual involvement with therapist: patient assesment, subsequent therapy, forensics. Washington DC.: American Psychological Association.

Pope, K.S., Sonne, J.L. \& Holroyd, J. (1993). Sexual feeling in psychotherapy. Explorations for therapists and therapists-in-training. Washington DC:
American Psychological Association.

Shernoff, M. (1995). AIDS: The therapist's journey. En M.B. Sussman (Ed.), A perilous calling: the hazards of psychotherapy practice (pp. 139-147). New York: John Wiley \& Sons.

Steinberg, M. \& Salamon, M.J. (1995). Geropsychology: how the venerable leave us vulnerable. En M.B. Sussman (Ed.), A perilous calling: the hazards of psychoterapy practice (pp. 45-54). New York: John Wiley \& Sons.

Stierlin, H. (1972). Self-actualization and philosophical awareness. En A. Burton (Ed.), Twelve therapists. San Francisco: Jossey-Bass, cit. in Sussman, M.B., o.c.

Strean, H.S. (Ed.) (1986). Countertransference. Current Issues in Psychoanalytic practice (Vol. 2) New York/London: Haworth Press.

Strean, H.S. \& Freeman, L. (1989). De jongen die me een machine noemde: Onthullingen van een psychoterapeut [Original: Behind the couch: relevations of a psychoanalyst]. New York: John Wiley \& Sons.

Sussman, M.B. (1992). A curious calling: unconscious motivations for practicing psychotherapy. Northvale, NJ/London: Jason Aronson.

Sussman, M.B. (Ed.) (1995). A perilous calling: the hazards of psychotherapy practice. New York: John Wiley \& Sons.

Tick, E. (1995). Therapists in the combat zone. En M.B. Sussman (Ed.), A perilous calling: the hazards of psychoterapy practice. (pp. 26-36). New York: John Wiley \& Sons. 


\section{Corveleyn}

Wolstein, B. (Ed.) (1988). Essential papers on countertransference. New York: New York University Press.

Yalom, I.D. (1989). Scherprechter van de liefde: Tien ware verhalen uit een psychotherapeutische praktijk [Original: Executioner and other tales of psychoterapy]. Amsterdam: Uitgeverij Contact. 